\title{
An Innovative Technique of Laparoscopic Knot Tying: A Qualitative Explanatory Research
}

\section{Forough Radfar ${ }^{1 *}$, Mohammad Zeinalddin ${ }^{2}$ and Moein Zeinalddin ${ }^{3}$}

${ }^{1}$ Senior Consultant and Head of Department of Obstetrics and Gynecology, Diwan of

Royal court Health Complex, Muscat, Oman

${ }^{2}$ Senior Lecturer and Course Coordinator, Oman Dental College, Muscat, Oman

${ }^{3}$ Oman Medical College, Muscat, Oman

*Corresponding Author: Forough Radfar, Senior Consultant and Head of Department of Obstetrics and Gynecology, Diwan of Royal Court Health Complex, Muscat, Oman.
Received: April 19, 2021

Published: May 25, 2021

(C) All rights are reserved by Forough Radfar., et al.

\section{Abstract}

Objective: To introduce an innovative laparoscopic knot tying technique using loop knot guide or needle knot guide designed for extracorporeal knot tying and using needle holder for intracorporeal knot tying to be ergonomically more suitable than traditional knot tying techniques for minimally invasive surgery.

Design: A qualitative, explanatory research design is used to describe a new technique of laparoscopic knot tying using our selfdesigned loop and needle knot guide devices for extracorporeal knot tying and using needle holder for intracorporeal knot tying.

Setting: Laparoscopic simulator trainer laboratory was used to examine different techniques of knot tying. No human or animal subjects were used.

Method: Various knot tying techniques (laparoscopic knots, surgical knots, knitting knots, fishing knots, and sailor's knots) were reviewed online using internet search engines, books and practical observation. The instruments which were used to assist in achieving knot tying with correct configuration, appropriate shaping and maximum security were studied.

We designed the new techniques of laparoscopic knot tying using our self-designed loop and needle knot guide and needle holder to cast the capsized form of the desired laparoscopic knot extracorporeally by working end of suture over them and pulling the standing end or needle end through it to form the sliding knot.

Technique: Intracorporeal knot tying: Working end of suture is casted in form of capsized configuration of the slip knot, on the shaft of needle holder before it is introduced into the body for intracorporeal suturing. Slip knot is formed by pulling the needle end of suture through the casted working end.

Extracorporeal Knot tying: Working end of suture is casted in form of capsized configuration of the slip knot, on the shaft of an appropriately sized self-designed loop or needle knot guide and the standing end is pulled through the casted working end of suture by using loop or needle knot guide, to form a slip knot extracorporeally. The knot is slid on standing end by knot pusher to the site where knot is to be applied.

Result: Using the innovative laparoscopic technique we achieved:

- Accurate knot reproducibility, security and stability.

- Least amount of suture material is used in forming the knot so it decreases risk of foreign body reaction in patient's tissues.

- Taking into consideration the ergonomics of laparoscopic surgery, this technique reduces fatiguability and trauma to surgeon 
- It also facilitates reduction of the angle between the working instruments while knot tying, so ports could be placed closer to each other, even single port laparoscopic knot tying is possible with this technique.

- It reduces instrument movements intracorporeally while applying the knot hence it decreases risk of trauma to patient.

Conclusion: Our innovative laparoscopic knot tying technique is an ergonomically suitable and simplified technique for laparoscopic knot tying, reduces related trauma to surgeon and reduces iatrogenic effects to patients.

Keywords: Minimally Invasive Surgery; Minimal Access Surgery; Laparoscopy; Extracorporeal; Intracorporeal; Knot Tying; Tissue Approximation

\section{Introduction}

Laparoscopic knot tying is the most challenging skill a surgeon must master in transforming from laparotomy to laparoscopic surgery. Many advances and technologies like clips, staples, pre-tied suture loop, tissue adhesives, laser tissue welding, Harmonic, Ligasure, electro-cautery are used in laparoscopic operations to ease the tasks by decreasing the need for knot tying, yet no surgeon can feel confident in attempting laparoscopic surgery before mastering the laparoscopic knot tying and suturing techniques, because as the patient safety is at stake during operation, surgeon cannot exclusively depend on these technologies which have their own limitations in applied surgery and are more expensive and machine dependent.

Surgeons are taught that it is vital to perfect their techniques of knot tying as within the strength of their knots, lay the safety and lives of their patients. The important basic principles of achieving knots with correct configuration, appropriate shaping and maximum security and stability, are related to understanding knot tying techniques. As Clifford W. Ashley says in his book of "knots": "a knot is either perfect or hopelessly wrong, it is never nearly right". To create our innovative knot tying technique we studied many basic principals of laparoscopic knot tying and considered the specific difficulties that we aim to overcome by our new technique.

Generally, the tied knot has three parts (Figure 1):

1. Loop which embrace the tissue, vessel or the two edges of divided wound which needs to be approximated.

2. Knot- The knot is composed of a number of throws or winds stacked against each other.

3. Ears- i) Needle-end or post limb of suture and ii) short limb or working limb.

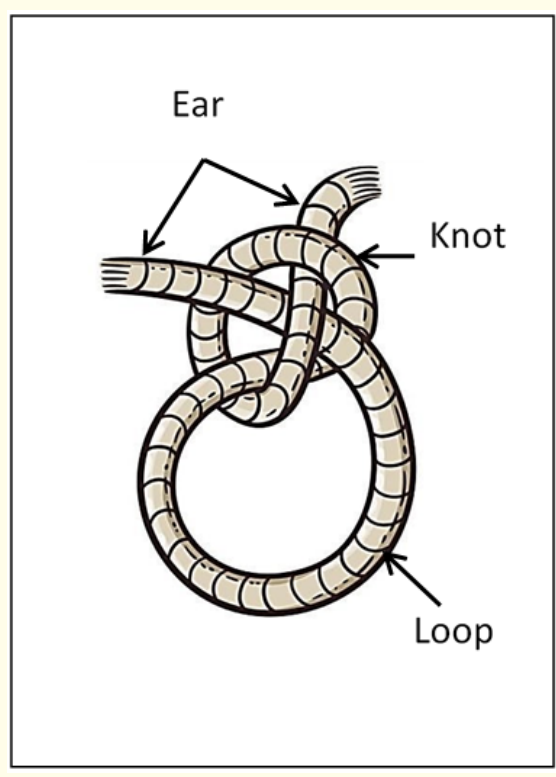

Figure 1: Parts of knot.

To understand the technique of knot tying we should know that to form an absolutely perfect knot we should concentrate in three stages of knot tying technique which are:

\section{Configuration}

Configuration means the composition of different components of knot such as throws, winds and half hitches forming the knot.

To avoid foreign body reaction to suture material, surgeon should choose the simplest knot configuration suitable for the situation, also the smallest suture size necessary to achieve strong tissue approximation should be used. The ears of knot should be cut as short as possible but not so short that knot can unravel. 
Factors affecting shaping of the knot are:

A. Tension applied on suture: The shape of knot made by each person is different. It is just like individual signature. If you might have noticed, if two people use same knitting needle and same yarn and switch while knitting you can easily tell the shape of knots differ even though they have applied the same knots configuration, it is because of the difference in tension applied on the yarn for stacking each component of knot on previous component (Figure 2a).

In surgical term keeping the components of knot in order is called "stacking". The tension applied by surgeon for staking each component of knot should be enough to make components of knot to stack appropriately. If excess tension is applied it might cause breaking of suture or cut the tissue. If less tension is applied while stacking, an insecure knot may form and in a surgical case, it may cause a catastrophe.

B. You might have noticed that in art of knitting, for different size threads different size needles or hooks are required. This is because if the size of the thread is not appropriate for diameter of the needle the knot will be too loose or too tight. If instruments are used to cast the components of knot on them for making the knot, for example where hooks or knitting needles are used, the diameter of the instrument should be appropriate for size of thread. The general rule in knitting is to use needles 2 - 3 times thicker than thread used in order to make a reasonably good knot [1]. The bigger the diameter of instrument, the looser the knots especially if more than two throws are used (Figure $2 b)$.

C. There is an important principle in one hand knot tying technique, "After tying the first half hitch, traction on standing end should be maintained to avoid loosening of loop before you make next throws". After we finish each consecutive throw and sliding it down to the knot, we must put traction on both ends equally to change the slip knot to flat knot configuration and hence change its character from sliding to non-sliding. If this step to change the sliding configuration to non-sliding flat knot configuration is not done, the knot may slip on standing end and loosen. This is the principal used in past pointing when sliding half hitches are pushed with knot pusher, to flatten the knot and prevent it from slipping.
In laparoscopic knot tying it is important to understand the details of changes in configuration of knot by changing the traction force from one end to another end of the suture.

D. Direction of traction force applied on each end of suture during knot tying, can bring about change in shape and geometry of knot:

a. Capsizing-Flat knot's geometry changes to slip knot geometry, for example overhand knot changes to half hitch by pulling one end (Figure 3 ). Some sliding knots are capsized version of flat knots for example Taut Line hitch and Nicky's knot are actually the capsized version of surgeons' knot [2] (Figure 4).

b. Flipping- Interchanging the traction between working end and standing end, relocate the knot from one end to the other (Figure 5). In case of sliding knot made up of half hitches, flipping changes the last half hitch position and make a reverse half hitch on alternating post. Flipping is useful in locking and securing the sliding knot.

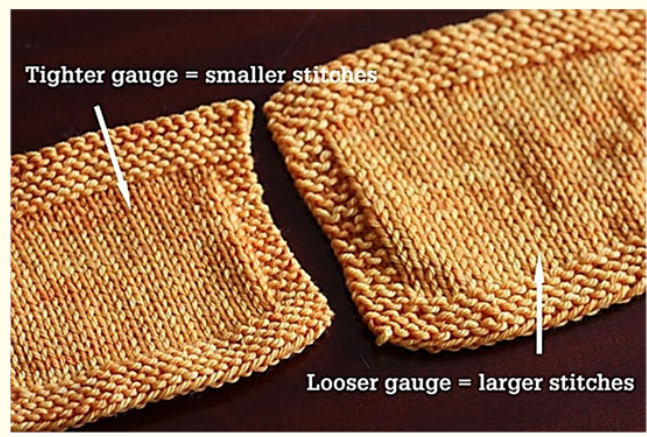

Figure 2a: Same size needle and same size thread were used.

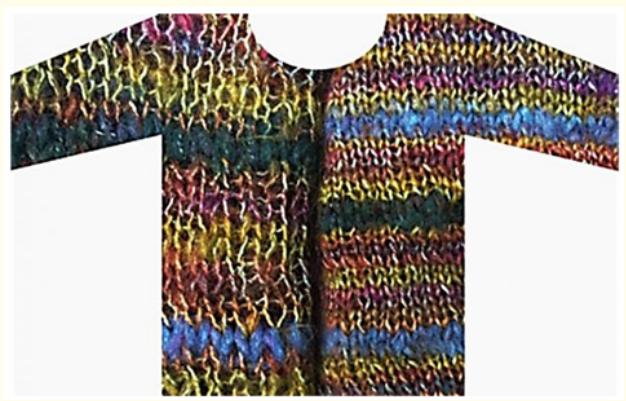

Figure 2b: Same size thread was used with different size needle. 


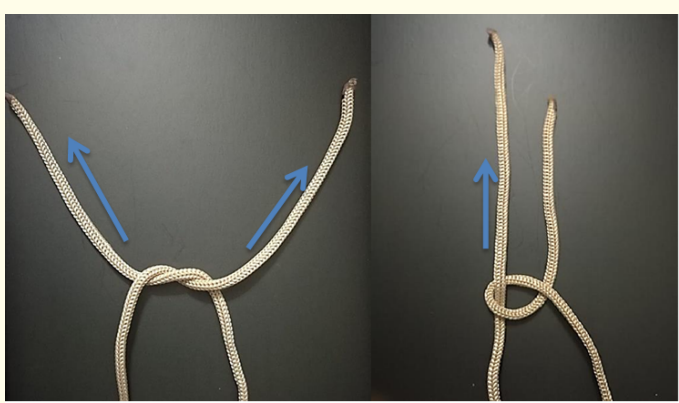

Figure 3: Capcizing-over hand flat knot changed to over hand sliding half hitch when the tension force is applied on post limb.

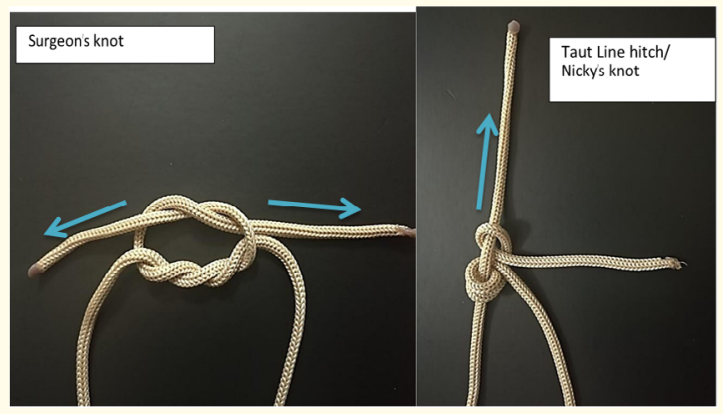

Figure 4: Taut line hitch/Nickys knot are actually the capsized version of surgeon's knot.

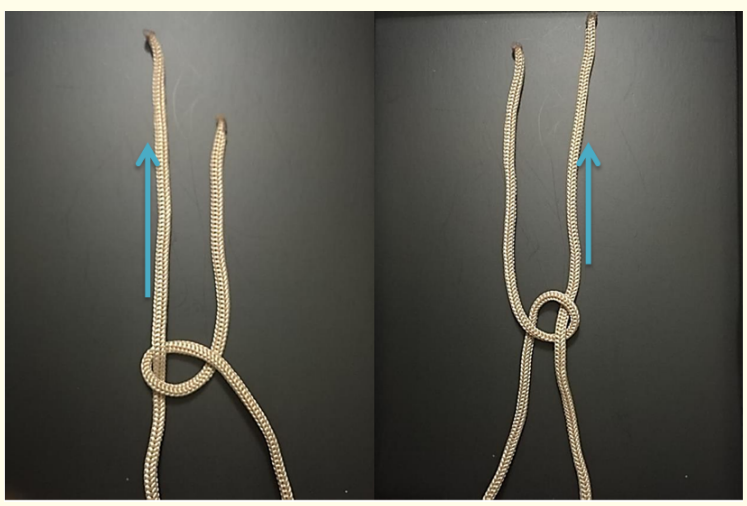

Figure 5: Flipping-interchanging the traction between working end and standing part, relocate the knot from one end to the other.

\section{Securing}

The completed knot must be secure, with no chance of slipping. We have to consider the following points in securing the knot:

a) Type of suture material used in knot tying, influences knot security. The braided sutures create more friction than monofilament sutures, so there is less possibility of knot loosening in braided compared to monofilament suture. The number of reverse half hitches for securing knots made by monofilament suture material need to be more, to increase length of contact and complexity between suture limbs so as to achieve higher internal interference and more knot security [3-6].

b) During laparotomy usually we use square knot and surgeons knot. We have learnt that applying few reverse half hitches will strengthen the security of the base knot. Some studies have shown, that under laboratory conditions, the ideal knot has 5 throws to maximize tensile strength and reduce the risk of untying. This finding does not seem to change with the type of suture material [7-9].

c) Most Studies have shown that addition of 3 RHAP in laparoscopic extracorporeal knot tying, improves knot security of all slip knots [3-6].

d) After each throw is made, for shaping a flat knot, both suture ends should be pulled to opposite direction, parallel to loop of knot, to secure the knot. In case of extracorporeal slip knots, past pointing with knot pusher will shape the half hitches into a flat knot.

e) To secure sliding knots, 3 RHAP are made. Each half hitch should be secured by changing its shape from sliding to flat knot using knot pusher past-pointing technique $[3,4,8,9]$.

Please note that after applying 3 RHAP, applying additional halfhitches, not only will not increase the security of knot, but also it increases tissue foreign body reaction and knot failure due to material failure (breakage) [3-6].

There are many laparoscopic ergonomic challenges during suturing and knot tying in addition to visual and tactile obstacles. Some examples are as follows: In laparoscopy the ports are fixed, and the freedom of movements is lost. The movement of instru- 
ments are around a fulcrum which is the port of entry; it restricts the movement of instruments to only 4 directions which are: rotation, up/down angulation, left/right angulation, in/out movement [10]. The working angle between two instruments should be more than 60 degrees, so the handles of instruments are further apart causing more tension on surgeon arms and shoulders and neck muscles while suturing and knot tying. During laparoscopy not only the surgeon's movements are restricted, but also all the muscles of arms shoulder, neck and back are activated and under tension, this causes lactic acid production in muscles and tendons and hence disabling muscle pain builds up, because of which the efficiency of surgeon reduces as the time of surgery increases [1012]. On the long run many surgeons have acquired permanent serious trauma such as headache [13], neck pain cervical spondylitis $[13,14]$, shoulder pain, finger joint pain in hands, tenosynovitis and hand muscle injury due to performing multiple laparoscopic procedures where suturing and knotting were required $[15,16]$.

When the handle of instrument is moved to any direction the tip of instrument moves in opposite direction due to fulcrum effect, it makes hand eye coordination difficulty.

Due to decoupling of visual axis and motor axis and two-dimensional perceptions during laparoscopic knot tying a lot of extra movements are made, for example, over shooting of instruments, these imprecisions of movements can cause damage to patient's tissues by the tip of instruments during the attempts to perform a laparoscopic knot. Imprecision of movement happens more in hands of less experienced laparoscopic surgeons.

Some common laparoscopic knot tying technical difficulties: The laparoscopic knot tying is classified under extracorporeal and intracorporeal techniques.

Techniques of intracorporeal knot tying such as expert 'c', Cinch, Smiley, Gladiator and Spaghetti techniques are aimed at forming a square knot, granny's knot or surgical knot as the base knot intracorporeally, followed by few reverse half hitches to secure it [17]. Recently some new techniques also have been introduced for laparo-endoscopic single-site surgery LESS, such as D-loop [18]. All these laparoscopic techniques aim to use the laparoscopic instruments to imitate surgeon's hands movement in knot tying.

There are also different extracorporeal knot tying techniques:

1. Non sliding (flat) knots, which are used when surgeon wants to avoid suture sliding through tissue or anchoring device.
The major drawback of intracorporeal knot tying and nonsliding extracorporeal knot tying is that the loop may loosen before second throw is placed on first throw to secure it.

In extracorporeal non-sliding knot tying, surgeon must note that if all the half hitches are made keeping the standing end on tension, the knot will have sliding property and is not locked safely. To achieve knot security the half hitches should be made on alternate posts or fastened by past pointing with knot-pusher to change the configuration of sliding knot to non-sliding knot.

2. Sliding or slip knots- which allow the knot to be tied extracorporeally and then it is slid on standing end, with a knot-pusher to the site where knot is to be applied. There are many types of slip knots such as: - Roeder knot, Duncan Knot, Nicky's knot, Weston knot, Meltzer knot, SMS knot, Tayside knot.

The main drawback of the sliding knot or slip knot tying technique is that its components should be stacked properly on the standing end otherwise its shape and configuration will change, as a result of this, either it will not slide on standing end or its loops are loose so they will form a faulty knot which does not have the property of intended slip knot. In addition, these loose knots allow more suture material to be left at surgical site causing more forging body reaction and increase chance of inflammation and infection. To make the proper configuration of slip knot using working end on the standing end using gloved hands during surgery needs a lot of practice and skill as both suture ends are thin, soft and highly mobile. In addition, gloved hands have less tactile sensation so these factors increase the chances of faulty sliding knot formation during laparoscopic extracorporeal knot tying.

Considering the above mentioned ergonomic and technical difficulties in laparoscopic suturing and knot tying, we conducted a qualitative, explanatory research on bases of which we introduce a new technique of laparoscopic knot tying, aiming at:

1) Reducing the movement of instruments intracorporeally while knot tying in order to reduce chance of trauma to tissues close to site of knot application.

2) Suitability to ergonomics of laparoscopic operative surgery, so as to reduce trauma to surgeon.

3) Simplifying accurate knot reproducibility, security and stability.

4) Reducing the amount of suture material used in knot, to decrease foreign body reaction in patient's tissues. 
Innovative technique of laparoscopic knot tying

This innovative technique of laparoscopic knot tying was tried several times in our lab using laparoscopic simulator, to make sure we can apply the laparoscopic knots with correct configuration, appropriate shaping and maximum security and stability.

\section{Instruments needed for knot tying:}

- 2 needle holders

- Maryland grasper dissector

- Knot pusher

- An artery forceps

- Laparoscopic scissors

- Loop or needle knot guide in case of extracorporeal knot tying.

We made the loop knot guide by bending the stylet of a spinal needle size 25 from mid-point over itself. (Figure 6). Thickness of the loop knot guide should be as close as possible to thickness of the suture used. If it is too thick the components of knot will be arranged very loosely on the standing end (faulty knot); if it is too thin, it cannot create enough space for the standing end to pass though the components of knot formed on it by the running end.

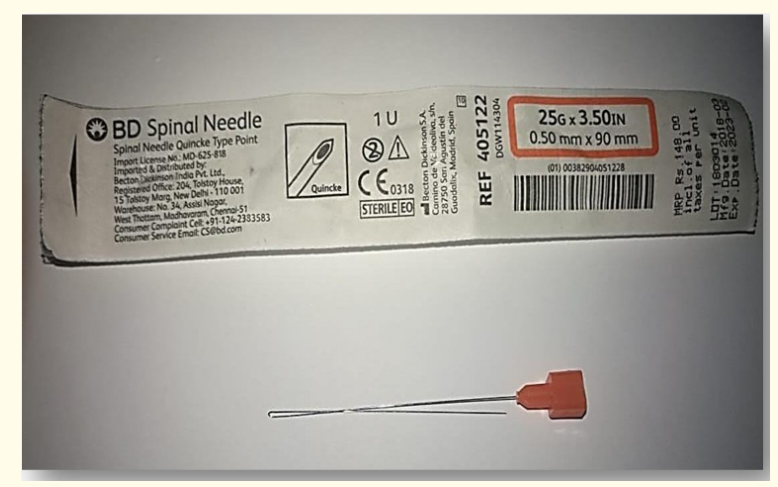

Figure 6: U-loop hook thread guide.

Needle knot guide-Sterile injection needle with smallest bore diameter to allow the standing end of suture to pass through its cavity can be used (Figure 7 and 8 ):

- $\quad$ Suture material.
- Metal rod reducer (size $7 \mathrm{~mm}$ ) - it should accommodate needle holder of $5 \mathrm{~mm}$ shaft diameter and should pass through port cannula of $10 \mathrm{~mm}$ diameter (Figure 9).

- Endoski needle is preferable. It is a needle which its proximal shaft of is 1.5 times the length of distal curved (1/4 of a circle) portion (Figure 10).

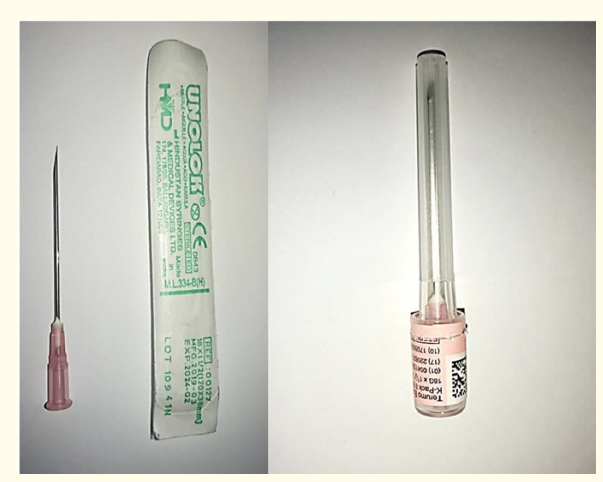

Figure 7: Needle thread guide.

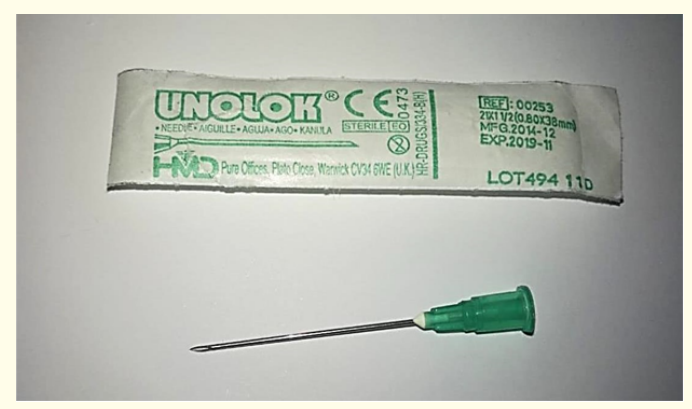

Figure 8: Needle thread guide.

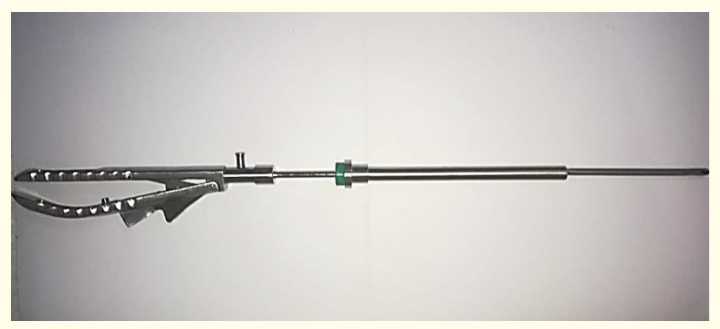

Figure 9: Pass the needle holder into metal reducer, so as to prevent distortion of the suture casted on needle holder while passing through the entry port. 


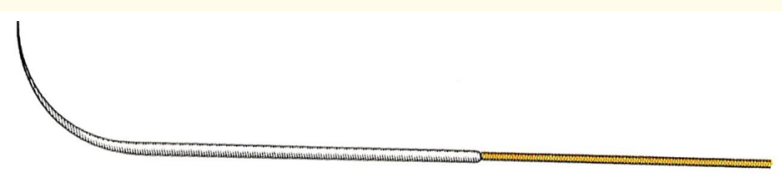

Figure 10: Endoski needle.

\section{Extracorporeal knot tying technique}

\section{Basic steps}

In this technique of extracorporeal knot tying, the standing end of suture is passed through the cavity of appropriate size needle guide or it is passed between the two prongs of the appropriate size loop guide, the working end or loop end of suture is casted on shaft of the loop guide or the Needle guide in form of capsized configuration of selected sliding/slip knot, the standing end is passed through the casted working end using loop or needle guide, to form a sliding knot.

The capsized configuration of flat knots such as Square knot, Granny's knot, surgeon's knot, Granny wise Surgeon's knot/Nicky's knot, Tennessee slider or What knot, which can be used as sliding knot are shown diagrammatically in figure 11.
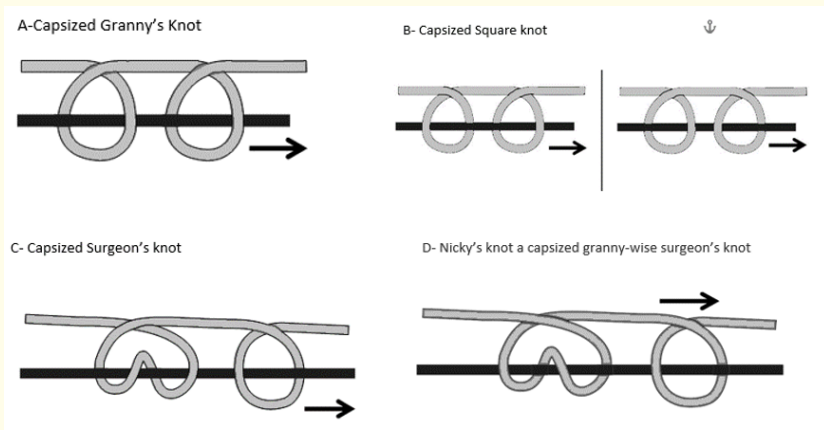

E-Tennessee slider, a capsized Whatknot

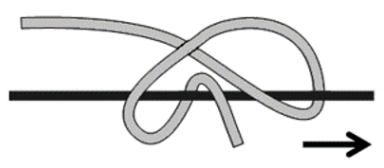

Figure 11
The capsized configuration of the compound sliding knots are shown diagrammatically in figure 12 .

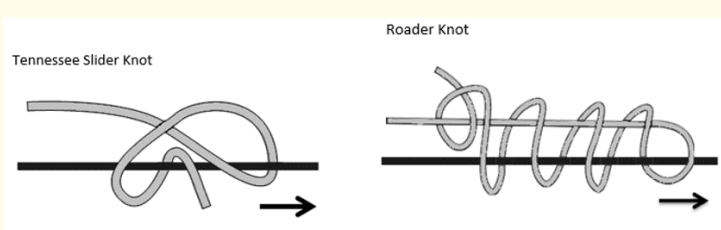

SMC knot

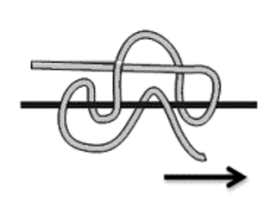

Weston Knot

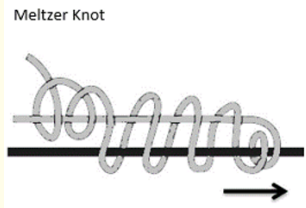

Tay-side Knot

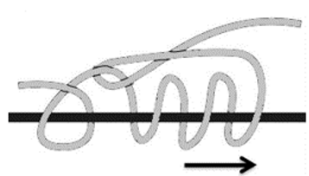

Figure 12

Compound Sliding knots can be divided into:

1. Non-locking such as Duncan loop which resists slipping by tightness of its wrappings of loop end around the post line [3].

2. Proximal locking such as Nicky's knot.

3. Middle locking knot such as SMC Knot, Tennessee slider knot [3].

4. Distal locking such as Weston knot, Roeder knot, Meltzer knot (specially was designed by Meltzer for PDS suture), Tayside knot (resistance to reverse slippage equivalent to surgeon's knot) [14].

The black line is used in diagrams to representing the guide (loop/needle knot guide) or standing end and the gray line represents the running end/loop end/working end of suture which is casted on the guide. The arrow indicates the direction of entry of the standing end through the components of knot formed by running end. 
After forming the sliding knot, it is shaped and then slid on the standing end by knot pusher to the site where the knot is to be applied as base knot. The sliding knots are then secured as explained before to prevent loosening of the knot $[3,4]$.

The number of the reverse half hitches applied to secure the sliding knot is decided according to the type of suture material, the type of base knot and the enforcement required in the particular situation by the surgeon.

Advantage of this technique over the traditional extracorporeal knot tying technique, is that forming the components of knot directly over standing end with working end of the thin suture material while wearing gloves during surgery is very difficult and there are high chances of imprecise knot formation. This method of using a metal needle or loop guide to form the capsized components of various knots on it, instead of directly forming the components of knot on the standing end as the traditional method, is very helpful during surgery to achieve appropriate stacking of components of knots over stiff guide so the sliding knots are made with precise and correct configuration extracorporeally. And as within the safety of our knots lies the safety of our patients, this is an exemplary method of extracorporeal knot tying.

Intracorporeal knot tying technique

\section{Basic steps}

A. Pass the needle holder into metal reducer, so as to prevent distortion of the suture, which would be casted on needle holder, while passing through the entry port canula (Figure 9).

B. First pass the needle holder though a Dundee Jamming loop which acts as an anchoring knot which prevent the basic knot loops from unraveling, then cast the required Flat knot capsized configuration (Figure 11) on the needle holder with working end of suture, around the shaft of needle holder, about $7 \mathrm{~cm}$ away from anchoring knot towards the grasping end of needle holder (Figure 13). Surgeon's knot provides the highest force to failure and the tightest loop circumference $[3,19]$ yet choice of the flat knot is according to surgeon decision and many surgeons use square knot.
(Note that, Dundee jamming loop does not participate in knot formation. After knot is applied, the short end is pulled and anchoring loop is opened and excess suture is trimmed).

C. In case Dundee Jamming knot, itself is to be applied, the shaft of needle holder is passed through Dundee jamming loop, after needle is passed through the tissue, the needle end of suture is then pulled through the loop to form the Dundee Jamming knot.

D. To introduce the needle and suture, hold the needle end of suture, one cm away from needle with needle holder. In case you want to make a free tie around a structure, hold the standing end, one and half $\mathrm{cm}$ away from its end with needle holder. Cover shaft of needle holder and needle suture complex within the metal reducer.

E. Introduce the needle holder and metal reducer which is covering casted suture on shaft of needle holder within it, into the $10 \mathrm{~mm}$ cannula entry port.

F. After the needle is passed through the tissue (or in case of making a knot around continuous structure, when needle holder passes under the structure which should be tied) the Maryland (or another needle holder) is used to hold the needle or standing end and pull the needle end of suture, then the needle end or standing end is passed on to needle holder by Maryland and the casted knot configuration formed by the short end or loop end of suture on needle holder shaft is slipped over the needle holder by Maryland so that the standing end held by needle holder passes through these components of knot to form a sliding knot (Figure 13). By pulling the standing end the slip knot slides to the tissue and tighten.

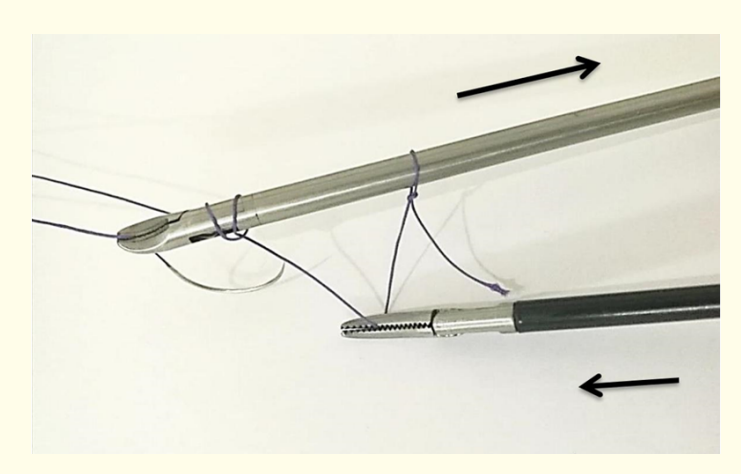

Figure 13 
Note that the standing end is not pulled through the anchoring Dundee jamming loop. After knot is applied, the Dundee jamming loop is slid off the needle holder shaft and the short end is pulled and anchoring Dundee Jamming loop is undone.

Then steps for shaping and securing the knot are performed. This capsized knot is secured from loosening or dismantling by flipping which means interchanging the tension from needle end of suture (standing end) to the short distal end of suture or loop end or working end; this will ideally change the shape of the knot, from sliding to a flat knot. By changing shape of the knot, the property of knot changes from a sliding knot to a strong non-sliding knot which is more secure. Then both sides of suture are pulled with equal force horizontally in opposite direction, to further tighten the base knot and prevent it from slipping or loosening.

Throwing few reverse half hitches, on this base knot will guaranty the security of knot from slipping or unwinding. The number of the reverse half hitches is decided according to the type of suture material and type of knot applied in the particular situation, by the surgeon.

\section{Discussion}

This innovative laparoscopic knot tying technique simplifies the intracorporeal and extracorporeal knot tying for surgeons, as it takes into consideration the ergonomics of laparoscopic operative surgery. Since all the components of base knot are casted outside the body i.e. on needle holder for intracorporeal knot tying, there is no need to have the working instruments at 60 degrees or more while knot tying. so even if ports are very close to one another or in single port laparoscopy, intracorporeal knot tying is possible using this technique. Moreover, the method is more suitable to ergonomics of laparoscopic operative surgery as compared to traditional method, so this fact reduces physical strain and fatigability and trauma to surgeon while operating.

The major disadvantage of conventional intracorporeal square or surgeon's knot tying and non-sliding extracorporeal square base knot tying is that the loop may loosen before second throw is placed on first throw to secure it. By using our innovative technique there is no chance of loop getting loose because capsized knot is slid to the site were square or surgeon's knot should be tied, the knot is secured by flipping it into flat knot. Furthermore, the time of intracorporeal knot tying is reduced as the components of flat knot is already casted on needle holder as its capsized form, so no need to perform two throws to make the flat knot.

Due to decoupling of visual axis and motor axis and two-dimensional perceptions during conventional intracorporeal knot tying a lot of extra movements are made, for example, over shooting of instruments, these imprecisions of movements can cause damage to patient's tissues by the tip of instruments during the attempts to perform a flat knot intracorporeally. Imprecision of movement happens more in hands of less experienced laparoscopic surgeons. Using the innovative intracorporeal knot tying these movements are reduced minimally even in hands of less experienced surgeons as the components of flat knot is already casted on needle holder as its capsized form, and it slides easily over standing end to site where it has to be applied, hence this new method reduces probability of injury to surrounding structures while performing intracorporeal knot tying.

Forming the components of knot directly over standing end with working end of the thin suture material while wearing gloves during traditional extracorporeal knot tying is very difficult and there are high chances of imprecise knot formation. Using this innovative method, the components of base knot are casted by working end on solid metal needle holder or loop/needle knot guide outside the body with more precision and faster and simplified compared to previous methods.

This method not only simplify accurate knot reproducibility, security and stability, but also it helps reducing the amount of suture material used in knot formation, to decrease foreign body reaction in patient's tissues.

This innovative method is an exemplary method of laparoscopic knot tying which enables Surgeons to master skill of laparoscopic knot-tying in shorter time as it has the above-mentioned advantages over the previous methods.

After applying the base knot, throwing few reverse half hitches, on the base knot will guaranty the security of knot from slipping or unwinding. The number of the reverse half hitches is decided according to the type of suture material and type of knot applied in the particular situation, by the surgeon.

Citation: Forough Radfar., et al. "An Innovative Technique of Laparoscopic Knot Tying: A Qualitative Explanatory Research". Acta Scientific Medical Sciences 5.6 (2021): 129-139. 


\section{Conclusion}

Innovative technique of laparoscopic knot tying is introduced taking into consideration:

- The ergonomics suitability to laparoscopic surgery.

- Decrease trauma to surgeon while operating laparoscopically and on the long term reduce disability of surgeons due to trauma acquired after many laparoscopic surgeries.

- Decrease trauma to patient tissues while applying the knot.

- Accurate Knot reproducibility, Knot security and stability.

- Least amount of suture material used in knot, to decrease foreign body reaction in patient's tissues.

- $\quad$ Fast and easy learning curve for surgeons learning laparoscopic knot tying.

\section{Disclosure of Interests}

Authors have no conflicts of interest.

\section{Contribution to Authorship}

All authors have contributed equally in the acquisition and analysis and interpretation of data regarding various knot tying techniques. We have worked together on conception of new laparoscopic knot tying technique. Drafting and revising of work critically and final approval has been additional responsibility of corresponding author.

\section{Ethics Approval}

This is not a clinical trial study hence no ethical approval was necessary.

\section{Funding}

This research has been funded by authors.

\section{Acknowledgement}

The Authors would like to acknowledge kind support of the Studies and Research Department of Directorate General of Medical Services of Diwan of Royal court of Sultanate of Oman.
Bibliography

1. Vermaire JH and van Exel N. "Parental attitudes towards oral health and caries-risk in their children". International Journal of Dental Hygiene 16.2 (2018): 241-248.

2. Baggio S., et al. "Early childhood caries in Switzerland: a marker of social inequalities". BMC Oral Health 15 (2015): 82.

3. Campus G., et al. "Changing trend of caries from 1989 to 2004 among 12-year old Sardinian children". BMC Public Health 7 (2007): 28.

4. Ferrazzano GF., et al. "Relationship Between Social and Behavioural Factors and Caries Experience in Schoolchildren in Italy". Oral Health and Preventive Dentistry 14.1 (2016): 55-61.

5. Nobile CG., et al. "Pattern and severity of early childhood caries in Southern Italy: a preschool-based cross-sectional study". BMC Public Health 14 (2014): 206.

6. Jordan AR., et al. "Early Childhood Caries and Caries Experience in Permanent Dentition: A 15-year Cohort Study". Swiss Dental Journal 126.2 (2016): 114-119.

7. Ccahuana-Vásquez RA., et al. "Effect of frequency of sucrose exposure on dental biofilm composition and enamel demineralization in the presence of fluoride". Caries Research 41.1 (2007): 9-15.

8. Ruel MT and Menon P. "Child feeding practices are associated with child nutritional status in Latin America: innovative uses of the demographic and health surveys". Journal of Nutrition 132.6 (2002): 1180-1187.

9. Fall CH., et al. "Infant-feeding patterns and cardiovascular risk factors in young adulthood: data from five cohorts in low- and middle-income countries". International Journal of Epidemiology 40.1 (2011): 47-62.

10. Thitasomakul S., et al. "Risks for early childhood caries analyzed by negative binomial models". Journal of Dental Research 88 (2009): 137-141. 
11. Schwarzer R., et al. "Stage-matched minimal interventions to enhance physical activity in Chinese adolescents". Journal of Adolescent Health 47.6 (2010): 533-539.

12. Orlando A., et al. "Can Nutrition Education in Primary Schools be an Efficient Way to Prevent Obesity in Adult Life? A Review of the Literature". Acta Scientific Nutritional Health 3.9 (2019).

13. National guidelines for the promotion of oral health and the prevention of oral diseases in developmental age, Ministero del Lavoro, della Salute e delle Politiche Sociali, Italia (2008).

14. National guidelines for the promotion of oral health and the prevention of oral diseases in developmental age, Update, Ministero della Salute, Italia (2013).

15. World Health Organization guidelines (2020).

16. Paglia L. "WHO: healthy diet to prevent chronic diseases and caries". European Journal of Paediatric Dentistry 19.1 (2018): 5.

17. Palacios C., et al. "Nutrition and health: guidelines for dental practitioners". Oral Disease 15.6 (2009): 369-381.

18. American Academy of Pediatrics. "Maintaining and improving oral health of young children". Pediatrics 134 (2014): 12241228.

19. Wagner Y., et al. "Evaluation of a regional German interdisciplinary oral health programme for children from birth to 5 years of age". Clinical Oral Investigation 21.1 (2017): 225-235.

20. Castilho AR., et al. "Influence of family environment on children's oral health: a systematic review". Journal of Pediatrics 89.2 (2013): 116-123.

21. Corrêa-Faria P., et al. "Incidence of dental caries in primary dentition and risk factors: a longitudinal study". Brazilian Oral Research 30.1 (2016): S1806-83242016000100254.

22. Clinical recommendations in odontostomatology, Ministero Della Salute, settembre (2017).

23. Karjalainen S. "Eating patterns, diet and dental caries". Dental Update 34.5 (2007): 295-298.
24. Ugolini A., et al. "Trends in Early Childhood Caries: An Italian Perspective". Oral Health and Preventive Dentistry 16.1 (2018): 87-92.

25. Contento IR. "Nutrition Education: Linking Research, Theory, and Practic. Burlington, MA Jones and Bartlett Learning". 3rd ed (2016).

26. Murimi MW., et al. "Factors that contribute to effective nutrition education interventions in children: a systematic review". Nutritional Review 76.8 (2018): 553-580.

\section{Volume 5 Issue 6 June 2021}

(C) All rights are reserved by Forough Radfar., et al. 